\title{
Robotic Enucleation of an Esophageal Submucosal Tumor in the Prone Position
}

\author{
Régis Souche, Jean-Michel Fabre \\ Chirurgie Digestive A, Département de Chirurgie Digestive, Hôpital Universitaire de St Eloi, Université de Montpellier, \\ Montpellier, France (both authors).
}

\begin{abstract}
Background: Esophageal submucosal gastrointestinal stromal tumors (GISTs) are rare. Resection is indicated due to their malignant potential.

Case Description: A 76-year-old woman complained of mild dysphagia. A submucosal tumor of the lower thoracic esophagus was found endoscopically. Computed tomography (CT) scan revealed a 40-mm anterolateral submucosal tumor. Endoscopic ultrasound revealed a hypoechoic and inhomogeneous lesion, developed from the fourth layer of the lower esophagus. In the prone position, the patient underwent robot-assisted right thoracoscopic enucleation of the esophageal submucosal tumor. The procedure was successfully completed with three trocars. No surgical complications occurred during the intervention or the postoperative stay. The patient was discharged on postoperative. Pathologic examination of the specimen revealed a GIST.

Conclusion: To the best of our knowledge, this is the first report of esophageal submucosal GIST enucleation with a patient in the prone position using robotic surgery platform. This approach, which complements the prone position by allowing a more precise dissection, is particularly appropriate for submucosal tumor enucleation and needs to be further evaluated.
\end{abstract}

Key Words: esophageal submucosal tumor, enucleation, robotic surgery, prone position, gastrointestinal stromal tumor.

Citation Souche R, Fabre J-M. Robotic enucleation of an esophageal submucosal tumor in the prone position. CRSLS e2014.00045. DOI: 10.4293/CRSLS.2014.00045.

Copyright $\odot 2014$ SLS This is an open-access article distributed under the terms of the Creative Commons Attribution-Noncommercial-ShareAlike 3.0 Unported license, which permits unrestricted noncommercial use, distribution, and reproduction in any medium, provided the original author and source are credited.

Address correspondence to: Régis Souche, MD, Chirurgie Digestive A, Département de Chirurgie Digestive, Hôpital Universitaire de St Eloi, Université de Montpellier, 80 avenue Augustin Fliche, 34295 Cedex 3 Montpellier, France. Telephone: +334 6733 70 72; Fax: +334 67 3370 84; E-mail: fr-souche@chu-montpellier.fr

\section{INTRODUCTION}

Gastrointestinal stromal tumor (GIST) is the most common mesenchymal tumor of the stomach and the intestine. However, esophageal submucosal GIST is rare compared with leiomyoma, which is more common. Recently, a mini-invasive approach with the prone position for esophageal surgery was thought to be an easier and safer procedure compared with the open approach. Here, we report a robotassisted thoracoscopy carried out with the patient in the prone position for enucleation of an esophageal submucosal tumor using the da Vinci robotic surgery platform.

\section{CASE DESCRIPTION}

\section{Patient}

A 76-year-old woman was referred to our center for a submucosal lesion of the lower esophagus. She had a past medical history of arterial hypertension, osteoporosis, and hypothyroidism. She also reported symptoms of gastroesophageal reflux disease with mild dysphagia. The physical examination was unremarkable, and her standard blood tests were within normal limits. Upper gastrointestinal endoscopy showed a normal mucosa with a near-obstructing lesion in the lower esophagus, easily crossed by the endoscope. Computed tomography (CT) scan revealed a $40 \times 30-\mathrm{mm}$ anterolateral submucosal tumor in the lower esophagus. This lesion was $8 \mathrm{~cm}$ above the esophageal hiatus and $5 \mathrm{~cm}$ below the aortic cross (Figure 1). There was no evidence of metastasis to the lungs or the liver. Endoscopic ultrasound revealed a hypoechoic and inhomogeneous lesion, developed from the fourth layer of the lower esophagus. An endoscopic ultrasound-guided fine needle aspiration performed before the patient was referred showed a GIST with Ki-67 index of less than $2 \%$. 
Robotic Enucleation of an Esophageal Submucosal Tumor in the Prone Position, Souche R et al.

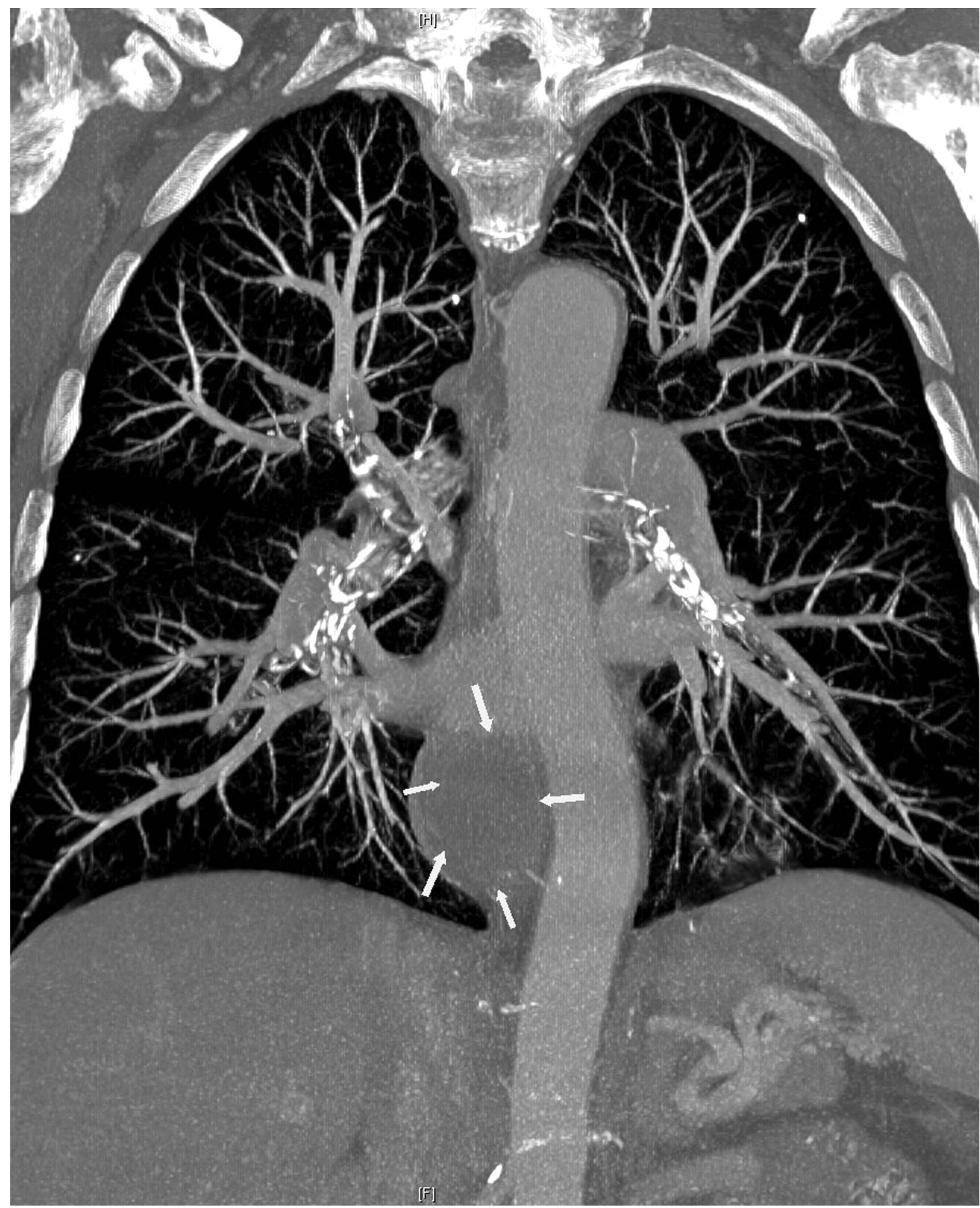

Figure 1. CT scan imaging showing a well-demarcated, $40 \times 30-\mathrm{mm}$ submucosal lesion of the lower esophagus. This lesion (shown by arrows) was $8 \mathrm{~cm}$ above the esophageal hiatus and $5 \mathrm{~cm}$ below the aortic cross. 
The patient's general condition and symptoms, as well as the lesion's histology and ultrasonographic appearance, indicated operative intervention.

\section{Operative procedure}

The patient underwent robot-assisted right thoracoscopic enucleation of the esophageal submucosal tumor. The patient was positioned in the prone position, with insertion of an armed single-lumen endotracheal intubation tube. The da Vinci S HD Surgical System (Intutive SurgicalSunny Valley-California) was positioned on the left side of the patient. Three trocars were used (fifth, seventh, and ninth intercostal spaces): one $12-\mathrm{mm}$ port for the $0^{\circ}$ scope and two 8-mm ports for the robotic arms on the posterior axillary line (Figure 2). $\mathrm{CO}_{2}$ was insufflated at a low pressure, set at a maximum of $8 \mathrm{~mm} \mathrm{Hg}$. In addition to the $0^{\circ}$ scope, only two instruments were used (monopolar cautery hook and bipolar forceps).

The mediastinal pleura on the right side of the esophagus was incised, with careful preservation of the main vagal trunk. After the submucosal tumor was localized, the overlying longitudinal and circular muscles were opened using hook cautery. Muscles were split longitudinally, and the plane between the tumor, muscularis propria, and underlying submucosa was developed. The lesion was separated from the muscular layer, while the underlying esophageal mucosa and submucosa were left intact (Figure 3). The integrity of the esophageal mucosa was confirmed by air insufflation and methylene blue test. Split muscular edges after the enucleation were not sutured. The specimen was inserted into a bag and extracted through the optical port site, which was expanded slightly to $2.5 \mathrm{~cm}$. A 20-French thoracic drain was placed inside the pleural space near the area of dissection, and no nasogastric tube was left.

The procedure was completed in 120 minutes, and blood loss was estimated at $25 \mathrm{~mL}$. The patient was extubated after the operation and did not require intensive care. The contrast medium swallow on postoperative day 2 showed good clearance of the esophagus and the absence of leaks or diverticula (Figure 4); hence, the patient was allowed a liquid diet. Pain medication was required for only the first two postoperative days (acetaminophen $4 \mathrm{~g}$ and tramadol $50 \mathrm{mg}$ ). The thoracic drain was removed on postoperative day 2 . The patient recovered uneventfully and was discharged on postoperative day 4. The patient returned to her normal daily activity and remained symptom-free at the 6-month follow-up. Pathologic examination of the specimen revealed GIST. The mitotic index was 3 mitoses per 50 high-power fields (HPFs) (focus 400). No adjuvant treatment was proposed due to the low recurrence risk according to Joensuu's 2011 classification. There was no sign of recurrence at the 6-month follow-up, during which a clinical review and CT scan were performed.

\section{DISCUSSION}

Submucosal tumors are rare lesions that account for less than $1 \%$ of all esophageal neoplasms. ${ }^{1}$ They are defined by their intramural and submucosal growth patterns. Esophageal GISTs represent approximately 10\% of all esophageal submucosal tumors. ${ }^{2}$ At histopathologic evaluation, immunohistochemical analysis is essential because GISTs, unlike leiomyomas, are at risk for recurrence, according to Joensuu's 2011 classification. 3,4 Preoperative

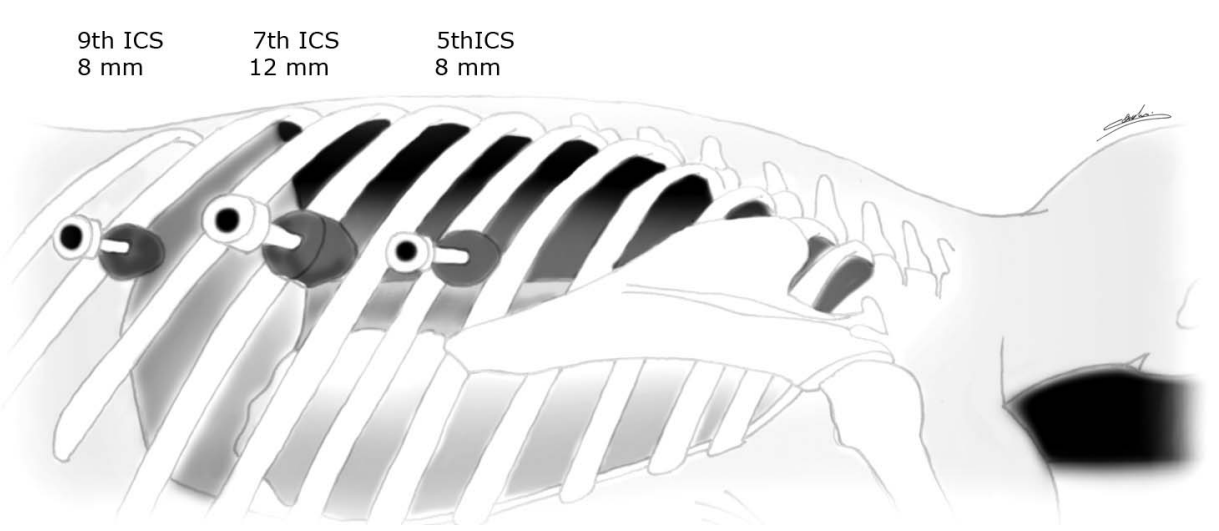

Figure 2. Patient in the prone position, with insertion of an armed single-lumen endotracheal intubation tube. The da Vinci $S$ system was positioned to the left side of the patient. Three trocars were used (fifth, seventh, and ninth intercostal spaces): one 12-mm port for the $0^{\circ}$ scope and two 8-mm ports for the robotic arms on the posterior axillary line. 
Robotic Enucleation of an Esophageal Submucosal Tumor in the Prone Position, Souche R et al.

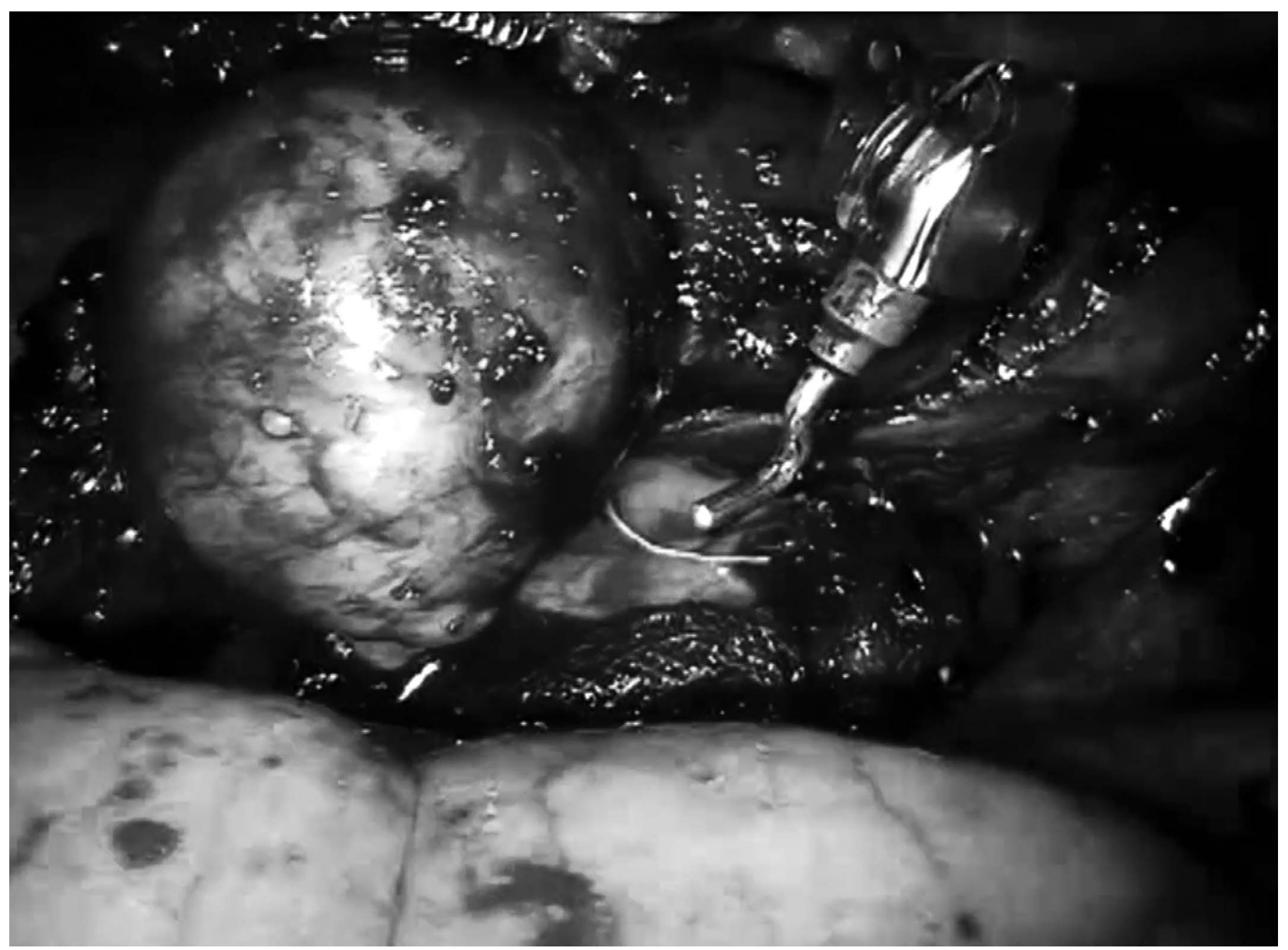

Figure 3. Submucosal tumor dissection from top to bottom.

biopsy of submucosal tumors is theoretically not indicated due to the risk of mucosal inflammation, which can complicate surgical enucleation and increase the risk of perforation of the mucosa..$^{5-7}$ However, when GIST is suspected, the size and especially the mitotic index of the lesion should be taken into consideration in choosing the best surgical approach and adjuvant therapy administration. Some authors recommend esophageal resection for lesions with a high mitotic index. ${ }^{8}$

The standard approach is open surgery via thoracotomy, which has been proven to be a safe procedure. ${ }^{9}$ Minimally invasive approaches, such as thoracoscopy, and later robotic surgery, have been successfully introduced into clinical practice. ${ }^{10,11}$

It is accepted that the thoracoscopic approach offers potential advantages compared with traditional thoracot- omy, including minimal aesthetic compromise and less pain. In addition, it is associated with better postoperative function, rapid and full reexpansion of the lung, lesser degree of pulmonary atelectasis, and lower incidence of pleural effusions. $5,10,12$

Considering the advantages of thoracoscopic enucleation of esophageal submucosal tumors, and the progress of thoracoscopy using the prone position, we proposed this technique using the da Vinci robotic surgery platform to our patient.

To date, only five case reports on the use of surgical robots in the management of esophageal submucosal tumors (leiomyomas) have been published, and no perioperative complications were reported. ${ }^{13-17}$ Thoracoscopic enucleation of an esophageal submucosal tumor, regardless of the histologic type, has been described in the prone 


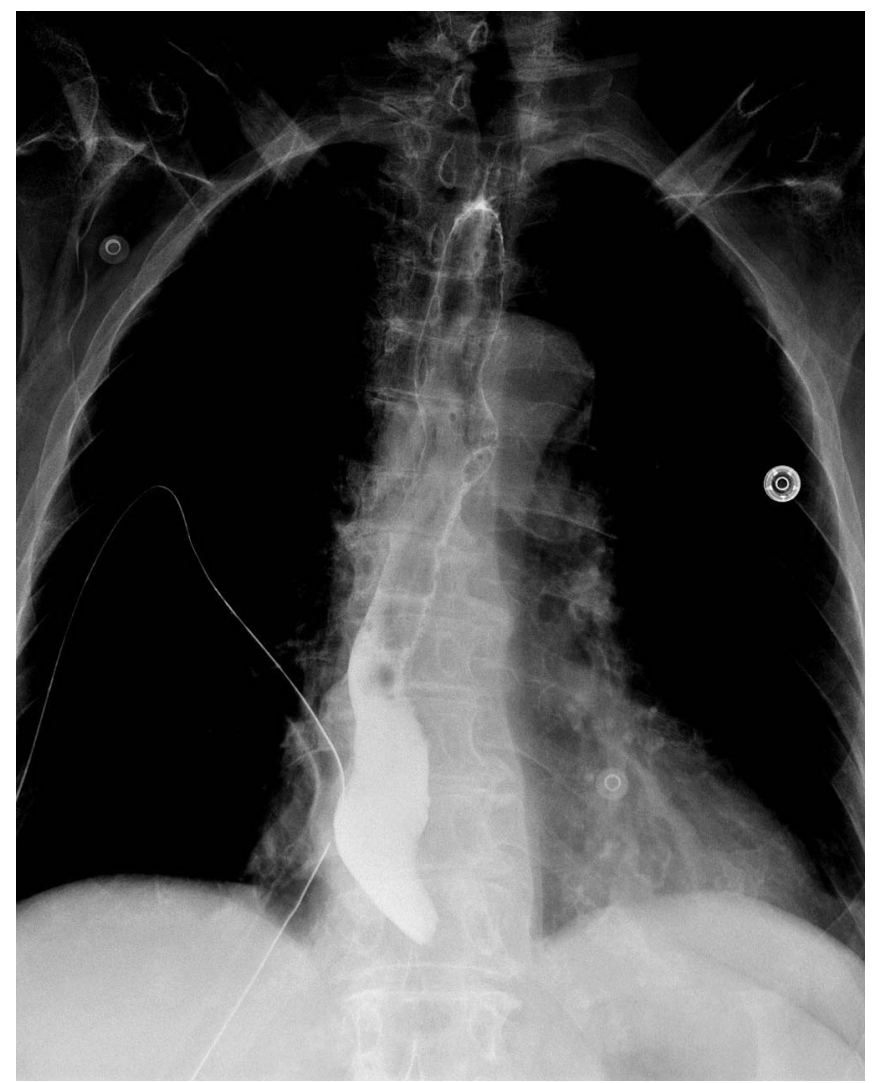

Figure 4. The contrast medium swallow on postoperative day 2 showed good clearance of the esophagus and the absence of leaks or diverticula.

position. ${ }^{18}$ However, the approach combining the prone position and robotic assistance has not been described.

The indications for robot-assisted thoracoscopic esophageal surgery are similar to those for classic thoracoscopic esophageal surgery. However, the robotic approach has additional advantages related to the three-dimensional view with high-definition image resolution. The articulated EndoWrist instruments, with several degrees of freedom, are useful in a confined operating field. They enhance dexterity and lead to a more precise dissection, particularly in the case of esophageal enucleation of GIST, in which tumor effraction and mucosal injuries must be avoided. The contraindications of the robotic approach would be previous thoracotomy on the same side and the existence of a circumferential esophageal tumor treated primarily by esophageal resection. ${ }^{10,19}$

The prone positioning technique is based on the principle that gravity will allow the cardiopulmonary block to fall anteriorly, allowing optimal exposure of the esophagus and facilitating dissection even in hemorrhagic cases. ${ }^{20-22}$ Another benefit of the prone position is that single-lung ventilation is not required, which simplifies the anesthetic procedure and allows a better ventilatory capacity.23,24 Several studies suggest that the prone position is physiologically well tolerated and may offer better oxygenation than the lateral decubitus or supine positions. ${ }^{25-27}$ In addition, due to the minimal trauma caused by this approach, there is no need to drain the pleural cavity systematically.

The time needed to place the patient in the prone position is not different from that needed to place the patient in the lateral position, and armed single-lumen endotracheal intubation saves times compared with double-lumen intubation for single-lung ventilation. However, the performance of robotic surgery requires an adequate learning curve, especially with regard to the docking step, the masters controls, and the lack of force feedback. Disadvantages of the prone position are the initial difficulty with the inverted anatomy and the conversion to open surgery by a posterior thoracotomy approach, which could be very complex, especially in the case of severe bleeding. ${ }^{19}$

In 2014, the main drawback of the robotic approach is its high cost, given that its perioperative outcome benefits compared with those of conventional laparoscopy have not been defined. The lack of competition in the surgical robots market, the surgeon's learning curve, and the inherent longer operative time contribute to this high cost. ${ }^{28}$ Robotic surgery could change the approach to submucosal esophageal tumors, especially GISTs, one of more conservative surgery. Further studies are required to evaluate if the benefit might justify the extra cost.

\section{CONCLUSION}

In conclusion, robot-assisted thoracoscopic enucleation of esophageal submucosal tumor is feasible and simple, and seems to be a safe procedure. Thoracoscopic surgery of the esophagus has already gained a wide acceptance by improving the postoperative recovery of patients. The prone position made the surgeons more meticulous by providing a clear operative field. The robot-assisted thoracoscopic approach complements the use of the prone position by facilitating a more precise dissection and minimizing the chances of mucosal perforation. Despite an increased cost compared with conventional laparoscopy, this approach deserves to be evaluated and seems particularly appropriate for submucosal tumor enucleation. 


\section{References:}

1. Seremetis MG, Lyons WS, deGuzman VC, Peabody JW Jr. Leiomyomata of the esophagus. An analysis of 838 cases. Cancer. 1976;38:2166-2177.

2. Portale G, Zaninotto G, Costantini M, et al. Esophageal GIST: case report of surgical enucleation and update on current diagnostic and therapeutic options. Int J Surg Pathol. 2007;15:393396.

3. Joensuu H, DeMatteo RP. The management of gastrointestinal stromal tumors: a model for targeted and multidisciplinary therapy of malignancy. Ann Rev Med. 2012;63:247-258.

4. Joensuu H, Hohenberger P, Corless CL. Gastrointestinal stromal tumour. Lancet. 2013;382:973-983.

5. Priego P, Lobo E, Rodriguez G, Cabanas J, Perez de Oteyza J, Fresneda V. Endoscopic treatment of oesophageal leiomyoma: four new cases. Clin Transl Oncol. 2007;9:106-109.

6. Zaninotto G, Portale G, Costantini M, et al. Minimally invasive enucleation of esophageal leiomyoma. Surg Endosc. 2006; 20:1904-1908.

7. Rijcken E, Kersting CM, Senninger N, Bruewer M. Esophageal resection for giant leiomyoma: report of two cases and a review of the literature. Langenbecks Arch Surg. 2009;394:623-629.

8. Peparini N, Carbotta G, Chirletti P. Enucleation for gastrointestinal stromal tumors at the esophagogastric junction: is this an adequate solution? World J Gastroenterol. 2011;17: 2159-2160.

9. Biere SS, Maas KW, Bonavina L, et al. Traditional invasive vs. minimally invasive esophagectomy: a multi-center, randomized trial (TIME-trial). BMC Surg. 2011;11:2.

10. von Rahden BH, Stein HJ, Feussner H, Siewert JR. Enucleation of submucosal tumors of the esophagus: minimally invasive versus open approach. Surg Endosc. 2004;18:924-930.

11. Palanivelu C, Prakash A, Senthilkumar R, et al. Minimally invasive esophagectomy: thoracoscopic mobilization of the esophagus and mediastinal lymphadenectomy in prone position-experience of 130 patients. J Am Coll Surg. 2006;203:7-16.

12. Akaraviputh T, Chinswangwatanakul V, Swangsri J, Lohsiriwat V. Thoracoscopic enucleation of a large esophageal leiomyoma using a three thoracic ports technique. World J Surg Oncol. 2006;4:70.

13. DeUgarte DA, Teitelbaum D, Hirschl RB, Geiger JD. Robotic extirpation of complex massive esophageal leiomyoma. J Laparoendosc Adv Surgl Tech A. 2008;18:286-289.

14. Elli E, Espat NJ, Berger R, Jacobsen G, Knoblock L, Horgan $\mathrm{S}$. Robotic-assisted thoracoscopic resection of esophageal leiomyoma. Surg Endosc. 2004;18:713-716.
15. Bodner JC, Zitt M, Ott H, et al. Robotic-assisted thoracoscopic surgery (RATS) for benign and malignant esophageal tumors. Ann Thorac Surg. 2005;80:1202-1206.

16. Ka-fung Chiu P, Wai-yan Chiu P, Yuen-bun Teoh A, Kinhung Wong S, Kwok-wai Ng E. Robotic-assisted thoracoscopic enucleation of esophageal leiomyoma. J Robotic Surg. 2011;5: 227-229.

17. Khalaileh A, Savetsky I, Adileh M, et al. Robotic-assisted enucleation of a large lower esophageal leiomyoma and review of literature. Int J Med Robot. 2013;9:253-257.

18. Yamada H, Shinohara T, Yokoyama K, Takasu K, Fujimori Y, Yamagishi K. Thoracoscopic enucleation of esophageal gastrointestinal stromal tumor using prone positioning in a patient with severe chronic obstructive lung disease. J Laparoendosc Adv Surg Tech A. 2011;21:635-639.

19. Claus CM, Cury Filho AM, Boscardim PC, Andriguetto PC, Loureiro MP, Bonin EA. Thoracoscopic enucleation of esophageal leiomyoma in prone position and single lumen endotracheal intubation. Surg Endosc. 2013;27:3364-3369.

20. Dapri G, Himpens J, Cadiere GB. Robot-assisted thoracoscopic esophagectomy with the patient in the prone position. $J$ Laparoendosc Adv Surg Tech A. 2006;16:278-285.

21. Cadiere GB, Himpens J, Dapri G. Esophagectomy by thoracoscopy in prone position followed by laparoscopy and cervicotomy. J Chir (Paris). 2006;143:237-242.

22. Shibasaki H, Kinoshita T, Ogata A, Miyazaki M. Thoracoscopic esophagectomy in the prone position. Hepatogastroenterology. 2012;59:1840-1843.

23. Gattinoni L, Protti A. Ventilation in the prone position: for some but not for all? CMAJ. 2008;178:1174-1176.

24. Richard JC, Bregeon F, Costes N, et al. Effects of prone position and positive end-expiratory pressure on lung perfusion and ventilation. Crit Care Med. 2008;36:2373-2380.

25. Pelosi P, Croci M, Calappi E, et al. The prone positioning during general anesthesia minimally affects respiratory mechanics while improving functional residual capacity and increasing oxygen tension. Anesth Analg. 1995;80:955-960.

26. Yatabe T, Kitagawa H, Yamashita K, Akimori T, Hanazaki K, Yokoyama M. Better postoperative oxygenation in thoracoscopic esophagectomy in prone positioning. J Anesth. 2010;24:803-806.

27. Jarral OA, Purkayastha S, Athanasiou T, Zacharakis E. Should thoracoscopic three-stage esophagectomy be performed in the prone or left lateral decubitus position? Interact Cardiovasc Thorac Surg. 2011;13:60-65.

28. Trehan A, Dunn TJ. The robotic surgery monopoly is a poor deal. BMJ 2013;347:f7470. 